\title{
Improvement in Rayleigh Scattering Measurement Accuracy
}

\author{
Amy F. Fagan* and Michelle M. Clem ${ }^{\dagger}$ \\ NASA Glenn Research Center, Cleveland, Ohio, 44135 \\ and \\ Kristie A. Elam \\ Jacobs Technology, Cleveland, $\mathrm{OH} 44135$
}

\begin{abstract}
[Abstract] Spectroscopic Rayleigh scattering is an established flow diagnostic that has the ability to provide simultaneous velocity, density, and temperature measurements. The Fabry-Perot interferometer or etalon is a commonly employed instrument for resolving the spectrum of molecular Rayleigh scattered light for the purpose of evaluating these flow properties. This paper investigates the use of an acousto-optic frequency shifting device to improve measurement accuracy in Rayleigh scattering experiments at the NASA Glenn Research Center. The frequency shifting device is used as a means of shifting the incident or reference laser frequency by $1100 \mathrm{MHz}$ to avoid overlap of the Rayleigh and reference signal peaks in the interference pattern used to obtain the velocity, density, and temperature measurements, and also to calibrate the free spectral range of the Fabry-Perot etalon. The measurement accuracy improvement is evaluated by comparison of Rayleigh scattering measurements acquired with and without shifting of the reference signal frequency in a 10 mm diameter subsonic nozzle flow.
\end{abstract}

\section{Nomenclature}

$d$

$=$ Fabry-Perot etalon thickness or plate spacing $(\mathrm{m})$

E $\quad=$ incident electric field vector $\left(\mathrm{V} \mathrm{m}^{-1}\right)$

$f \quad=$ focal length of a lens $(\mathrm{m})$

$f_{C} \quad=$ collimating lens focal length $(\mathrm{m})$

$f_{L} \quad=$ fringe forming lens focal length $(\mathrm{m})$

$I_{F P} \quad=$ Fabry-Perot instrument function

$\mathbf{K}=$ interaction wave vector $\left(\mathrm{m}^{-1}\right)$

$\mathbf{k}_{\mathbf{0}} \quad=$ incident light wave vector $\left(\mathrm{m}^{-1}\right)$

$\mathbf{k}_{\mathbf{s}} \quad=$ scattered light wave vector $\left(\mathrm{m}^{-1}\right)$

$r \quad=$ radial position in image plane $(\mathrm{m})$

$T=$ static temperature $(\mathrm{K})$

$u_{k} \quad=$ measured velocity component along $\mathbf{K}$ direction $\left(\mathrm{m} \mathrm{s}^{-1}\right)$

$\beta \quad=$ angle between $\mathbf{E}$ and scattering plane (rad)

$\varepsilon \quad=$ optical system efficiency

$\theta \quad=$ angle of the light ray passing through etalon (rad)

$\lambda=$ illumination wavelength $(\mathrm{m})$

$\mu \quad=$ etalon cavity refractive index

$v \quad=$ optical frequency $\left(\mathrm{s}^{-1}\right)$

$v_{0} \quad=$ optical frequency of laser output $\left(\mathrm{s}^{-1}\right)$

$\rho \quad=$ gas density $\left(\mathrm{kg} \mathrm{m}^{-3}\right)$

$\chi_{s} \quad=$ scattering angle (rad)

\footnotetext{
${ }^{*}$ Research Engineer, Optical Instrumentation and NDE Branch, 21000 Brookpark Road, AIAA Senior Member

${ }^{\dagger}$ Research Engineer, Optical Instrumentation and NDE Branch, 21000 Brookpark Road

${ }^{\ddagger}$ Optics Technician, 21000 Brookpark Road
} 


\section{Introduction}

$\mathrm{R}_{\mathrm{t}}$ AYLEIGH scattering is the elastic scattering of light from molecules where the signal strength, Doppler frequency shift, and spectral linewidth of the scattered light provide measurements of density, velocity, and temperature, respectively ${ }^{1}$. Typical Rayleigh linewidths are on the order of 1-2 GHz; hence a very narrow linewidth laser $(\sim 5 \mathrm{MHz})$ and an extremely high resolution filter are required to resolve the spectrum. These high resolution filters can be atomic or molecular absorption filters (filtered Rayleigh scattering ${ }^{2,3}$ ) or filters based on interference phenomena, such as the Fabry-Perot (FP) interferometer (interferometric Rayleigh scattering (IRS)). A FP interferometer ${ }^{4}$ consists of two parallel planar reflective plates or surfaces and is used in the imaging mode (constant spacing between reflective surfaces) for this IRS implementation. When the FP interferometer has fixed plate spacing, it is often called an etalon. An etalon may be air-spaced or consist of a solid transparent optical material. When light is imaged through the etalon an interference pattern results which is a function of the spectrum of the light convolved with the instrument function of the $\mathrm{FP}^{4}$, which is the Airy function.

Using an optical fiber to collect the Rayleigh scattered light provides point-wise or spatially-averaged measurements over a finite distance (on the order of 0.5-1.0 mm) along a focused laser beam. Our research group at the NASA Glenn Research Center (GRC) has developed several fiber-coupled point-wise Rayleigh scattering measurement systems that are capable of acquiring data at high sampling rates using high quantum efficiency detectors $^{5-7}$. Multiple-point, spatially-resolved measurements have also been obtained by imaging scattered light from a laser line or sheet directly through a FP interferometer ${ }^{8-11}$. A direct imaging type of system requires that the interferometry equipment be located in or near the facility. An air-spaced Fabry-Perot is usually too delicate to operate in locations with high vibrations such as experienced in most NASA test facilities; therefore a solid etalon is typically used. One of the largest sources of velocity measurement error comes from laser frequency drift when a reference frequency measurement is not acquired at the same instant as the Rayleigh scattering signal. The velocity measurement error associated with uncertainty in the laser frequency is approximately $0.4 \mathrm{~m} / \mathrm{s} \mathrm{per} \mathrm{MHz}$. Typical frequency drift observed in the Rayleigh work at NASA GRC is $5-20 \mathrm{MHz}$, giving velocity errors of $2-8 \mathrm{~m} / \mathrm{s}$. In many Rayleigh scattering experiments utilizing a CCD camera to detect the interference fringe pattern a small amount of incident laser light is spectrally analyzed along with the Rayleigh scattered light to provide a simultaneous reference of the incident laser signal, thereby eliminating the velocity measurement uncertainty attributable to laser frequency drift ${ }^{11-13}$. However, when this is done there is often significant overlap of the Rayleigh and reference fringes in the image because the frequency shift between the two signals is not large enough to distinctly separate the two fringes. In this paper an acousto-optic frequency shifting device is used to artificially shift the reference laser signal by $1100 \mathrm{MHz}$ in an attempt to overcome this problem and improve the measurement accuracy. The frequency shifting device is also used as a tool to calibrate the free spectral range (FSR) of the solid etalon. The proof-of-concept experiment presented in this paper is performed in a small bench top free jet. Eventually, the improved Rayleigh system is targeted for use in ground test facilities such as the Aero-acoustic Propulsion Laboratory and the $15 \mathrm{~cm} \times 15 \mathrm{~cm}$ Supersonic Wind Tunnel at NASA GRC.

\section{Rayleigh Scattering and Fabry-Perot Interferometry}

In molecular Rayleigh scattering an incident electric field interacts with an atom or a molecule inducing a dipole moment that oscillates and radiates at the frequency of the incident field. It is considered an elastic scattering process because the internal energy of the molecule is unchanged and the frequency of the light is changed only by the Doppler effect due to the thermal as well as the bulk motion of the molecules ${ }^{1}$. The frequency spectrum of the scattered light contains information about the gas density, bulk velocity, and temperature. Figure 1 shows a Rayleigh scattering spectrum containing the narrow laser line and a typical Rayleigh spectral peak to illustrate how the flow property measurements are obtained from the spectral information. If the gas composition is fixed, the total intensity of the Rayleigh scattered light is directly proportional to the gas density $\rho$. The frequency shift between the laser peak and the Rayleigh peak is proportional to the bulk flow velocity $u_{k}$. The width of the spectrum is related to the gas temperature $T$. The measured velocity component, $u_{k}$, is in the same direction as the interaction wave vector $\mathbf{K}$, which is the bisector of the incident and scattered light wave vectors, $\mathbf{k}_{\mathbf{0}}$ and $\mathbf{k}_{\mathbf{s}}$, respectively, as shown in Fig. 2. The scattering angle $\chi_{\mathrm{s}}$ is defined relative to the incident light wave direction. The geometry of the optical arrangement in an experiment is designed such that the desired component of the velocity vector is measured. Experiments are typically arranged such that the electric field vector $\mathbf{E}$ is perpendicular to the scattering plane defined by the incident and scattered light wave vectors $\left(\beta=90^{\circ}\right.$, 's'-type or vertical polarization (Fig. 2)). In this configuration the collected scattering intensity is independent of the scattering angle and maximized. 


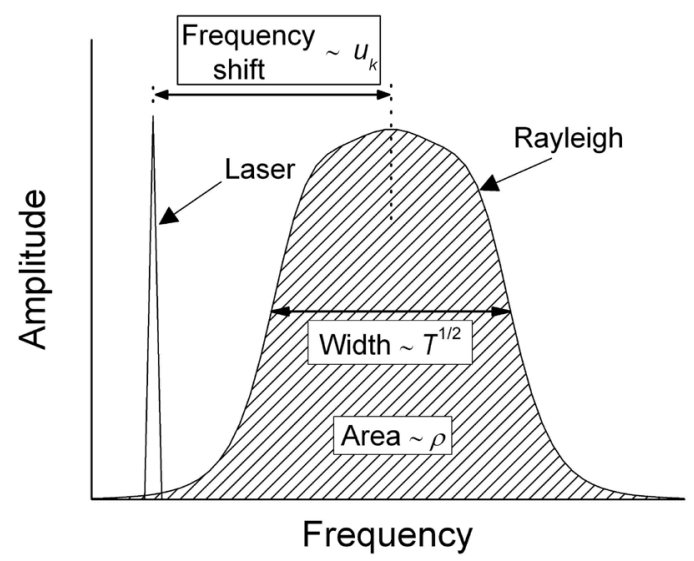

Fig. 1 Rayleigh scattering spectrum.

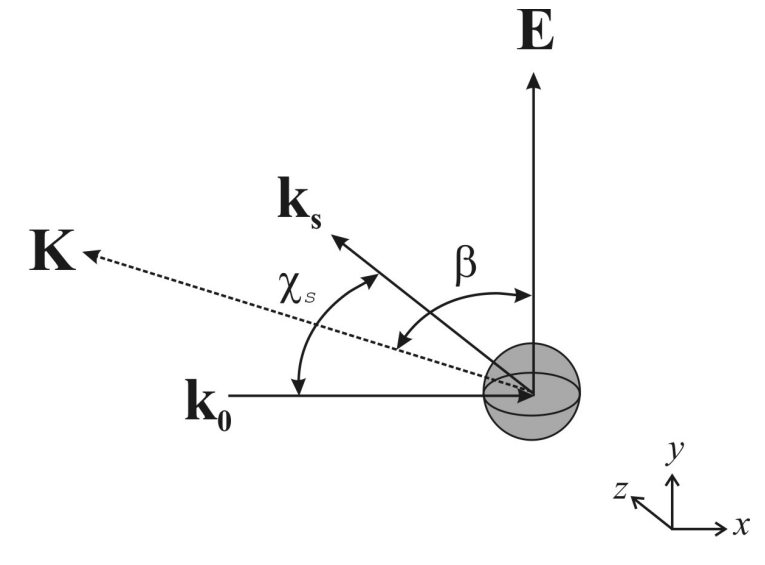

Fig. 2 Light scattering from a moving particle.

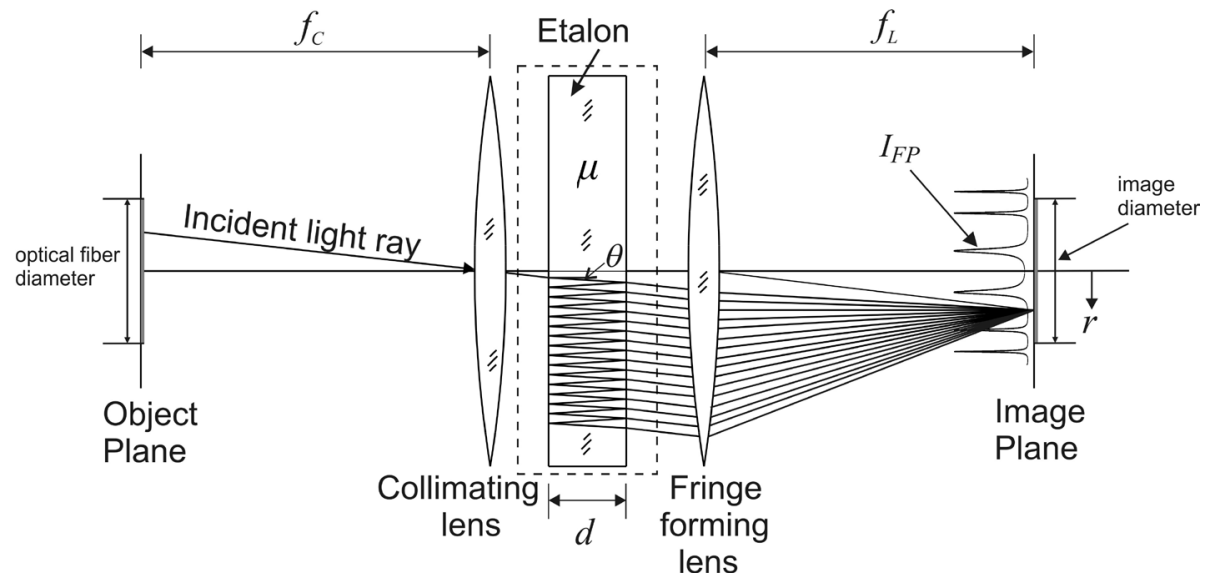

Fig. 3 Basic optical arrangement for spectrally-analyzing a uniform light source using a solid Fabry-Perot etalon.

A Fabry-Perot interferometer or etalon is commonly used to resolve the Rayleigh spectrum. A detailed discussion of the theory on FP interferometry as applied to this type of Rayleigh scattering experiment can be found in Reference 14. A common arrangement for spectrally-analyzing Rayleigh scattered light using a planar FabryPerot interferometer is shown in Fig. 3 where light from the object plane (an optical fiber output, in this case) is imaged through the etalon and imaged at the image plane which is typically a CCD detector. A typical interference fringe pattern that results at the image plane when the object is a planar, uniform, single-frequency light source is shown in Fig. 4. The resulting interference pattern is the spectrum of the light convolved with the instrument function of the Fabry-Perot, which is the Airy function. For spectrally broadened light, such as Rayleigh scattered light, the fringes broaden accordingly; the linewidth of the fringes provides a measure of temperature. The frequency shift of the light, which is associated with the bulk flow velocity, is determined by a spatial shift in the fringe positions in the image.

In the current experiment Rayleigh scattered light is collected by an optical fiber from a finite length of the laser beam and imaged through the solid FP etalon onto a CCD detector. A small amount of incident or reference laser light is also input into the signal fiber and imaged with the Rayleigh scattered light. An acousto-optic frequency shifting device is used to induce a known shift in the reference laser frequency to spatially shift the reference fringes away from the Rayleigh fringes in the interference pattern and improve the accuracy of the measurements since the reference and Rayleigh fringes will no longer overlap. Figure 5 shows unshifted incident laser light (innermost of the two fringe sets) and the incident laser light shifted up in frequency by $1100 \mathrm{MHz}$ (outermost of the two fringe sets). The Rayleigh signal in the experimental configuration implemented here is Doppler shifted from the incident 
laser frequency by a much smaller amount, on the order of 10's to 100's of $\mathrm{MHz}$ and in the opposite direction. Figure 6 shows the lowest order interference fringe of a typical Rayleigh signal (velocity=190 m/s) coupled with unshifted reference laser signal showing that the two fringe peaks overlap one another. A line profile through the fringe shown to the left of the image demonstrates more clearly how difficult it is to discern the two individual peaks. Figure 7 shows a Rayleigh fringe having the same flow conditions as in Fig. 6 coupled with reference laser signal that has been artificially shifted by $1100 \mathrm{MHz}$ using the acousto-optic frequency shifting device. Comparing the line profile of Fig. 7 with that of Fig. 6 it is obvious to see the benefit of implementing an artificial frequency shift on the reference signal. The Rayleigh peak can clearly be differentiated from the reference signal peak in Fig. 7, enabling the Rayleigh and shifted reference fringes to be more accurately evaluated in the modeling of the fringe pattern. The theoretical model of the fringe pattern containing both reference laser light and Rayleigh scattered light has been developed and presented previously ${ }^{14}$. The Rayleigh spectrum in the model is evaluated using the TENTI S6 kinetic theory mode ${ }^{15,16}$. The imaged fringe patterns are analyzed by maximum likelihood estimation ${ }^{17}$ using the developed model function described in Eq. (11) of Ref. 14 to evaluate the density, temperature, and axial velocity at a single spatial point in the flow field. Data is acquired in the jet core of a subsonic $10 \mathrm{~mm}$ diameter nozzle flow to demonstrate the improvement in measurement accuracy.

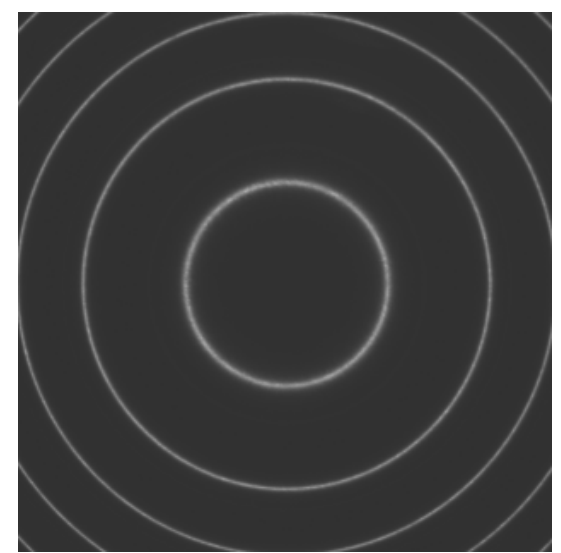

Fig. 4 Concentric ring interference pattern from a planar single-frequency light source imaged through a Fabry-Perot etalon.

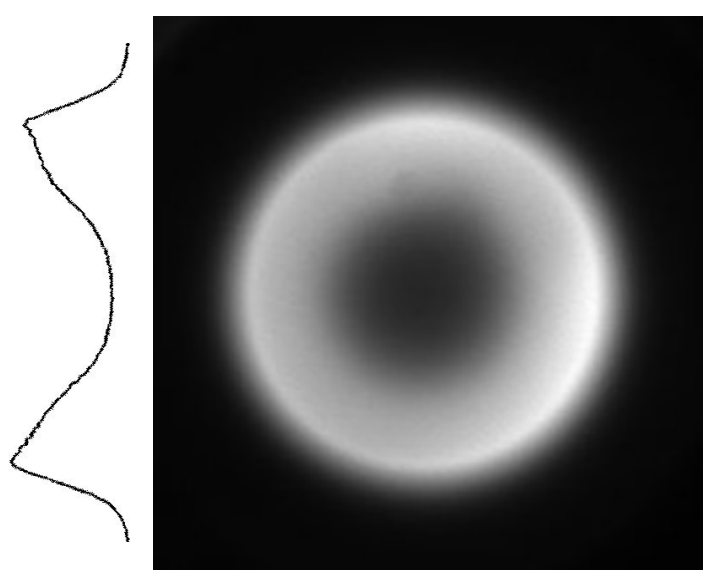

Fig. 6 Rayleigh signal for $190 \mathrm{~m} / \mathrm{s}$ flow (inner fringe) and unshifted reference laser signal (outer fringe) imaged through a Fabry-Perot etalon. A line profile through the center of the fringe is shown to the left of the image.

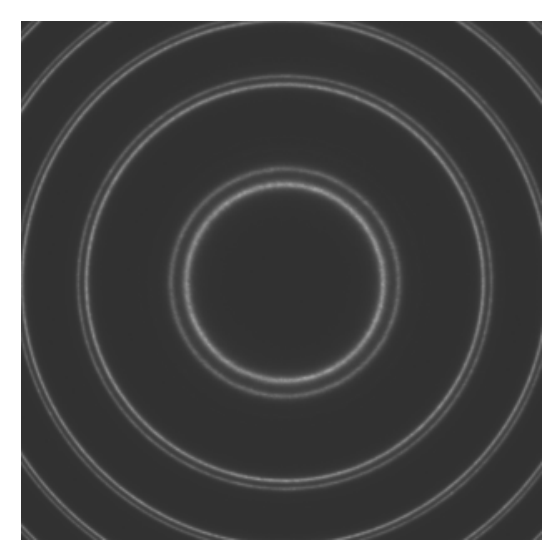

Fig. 5 Concentric ring interference pattern from two single-frequency sources with a frequency difference of $1100 \mathrm{MHz}$ imaged through a Fabry-Perot etalon.

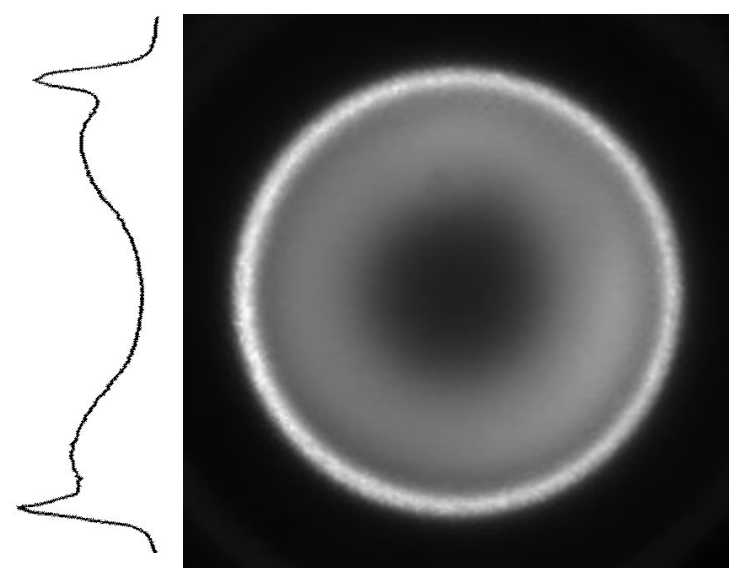

Fig. 7 Rayleigh signal for $190 \mathrm{~m} / \mathrm{s}$ flow (inner fringe) and reference laser signal artificially shifted by 1100 MHz (outer fringe) imaged through a Fabry-Perot etalon. A line profile through the center of the fringe is shown to the left of the image. 


\section{Experiment}

Rayleigh scattering data is acquired in a subsonic flow exiting a small bench top free jet equipped with a $10 \mathrm{~mm}$ diameter convergent nozzle for this proof-of-concept experiment. A top-view diagram of the Rayleigh scattering system is shown in Fig. 8. A Coherent Verdi $18 \mathrm{~W}$ continuous-wave $532 \mathrm{~nm}$ laser with a $2.25 \mathrm{~mm}$ diameter output beam and $5 \mathrm{MHz}$ linewidth provides the incident light for the system. A half-wave plate is used to rotate the polarization of the beam to orient the electric field vector perpendicular to the scattering plane at the probe volume in order to maximize the power of the scattered light in the direction of the collection optics. The laser beam is focused with a $175 \mathrm{~mm}$ focal length lens (L1) to a $1 / \mathrm{e}^{2}$ diameter of $50 \mu \mathrm{m}$ at the probe volume. The beam is oriented at a $45^{\circ}$ angle to the primary flow direction, while the scattered light is collected at a $90^{\circ}$ angle relative to the incident laser beam. The incident and scattering wave vectors are arranged such that the negative axial component of the jet velocity is measured as indicated by the red $\mathbf{K}$ vector in Fig. 8. A finite length of the laser beam is imaged by a pair of $f / 4200 \mathrm{~mm}$ focal length lenses (L2 and L3) onto the face of a $0.91 \mathrm{~mm}$ diameter multimode optical fiber, shown in blue in Fig. 8. Since the lenses provide one-to-one imaging, the probe volume length is $0.91 \mathrm{~mm}$ along the laser propagation direction and the cylindrical probe volume diameter, which is set by the focused beam waist, is $50 \mu \mathrm{m}$. The measurement probe volume is located within the core of the filtered air nozzle flow along the flow axis at an axial distance of two jet diameters from the nozzle exit.

At the output of the laser a beamsplitter in the optical path sends $10 \%$ of the reference laser light to the acoustooptic frequency shifting device, which shifts the incident laser frequency up by $1100 \mathrm{MHz}$ with absolute accuracy of $0.01 \%$. The acousto-optic device produces two output beams: an unshifted and shifted beam; the beams are separated by an angle equal to twice the Bragg angle associated with the internal Bragg cell. The unshifted beam is blocked by a beam dump (BD1). The shifted reference beam is focused and collected into a multimode fiber, referred to as the reference fiber (indicated in red in Fig. 8), that transmits the shifted reference light to the signal collection fiber (indicated in blue in Fig. 8). A small amount of the shifted reference laser light is coupled into the signal fiber by a $50 \mathrm{~mm}$ focal length lens (L4). This configuration allows reference and Rayleigh signals to be imaged and recorded together, providing a simultaneous reference of the incident laser frequency and removing any uncertainty or bias error due to lack of information about the incident laser frequency at the time of the Rayleigh signal data acquisition as was observed in previous experiments ${ }^{18}$. Image data are also acquired with unshifted reference light for comparison purposes to evaluate the accuracy improvement gained by inducing an artificial shift in the reference signal. In this case the unshifted output from the acousto-optic device is collected into the reference fiber while the shifted beam is directed to the beam dump.

The signal fiber transmits the collected signals to another optical table located in close proximity to the collection optics. The light exiting the fiber is collimated by a $60 \mathrm{~mm}$ focal length lens (L5) and is directed through the etalon. The $25 \mathrm{~mm}$ diameter fused silica etalon has a refractive index of approximately 1.46 at room temperature and the $90 \%$ reflectivity coatings provide a reflective finesse of 30 . The actual finesse is about half of the reflective finesse value due to surface quality and tilt error. The etalon is approximately $11.2 \mathrm{~mm}$ thick resulting in a FSR of approximately $9 \mathrm{GHz}$. The exact FSR is calibrated using shifted and unshifted reference light imaged simultaneously through the etalon as shown in Fig. 5. To do this, a third optical fiber (not shown in Fig. 8) replaces BD1 and is used to collect unshifted reference signal and is simultaneously coupled into the signal fiber along with the shifted reference signal using another $50 \mathrm{~mm}$ focal length lens. The Rayleigh signal is blocked in this case so that only the two reference signals are transmitted to the Fabry-Perot etalon and imaging equipment. The known frequency shift between the two signals is used to evaluate the FSR of the etalon. Obtaining an accurate measure of the FSR of the etalon improves the ability to model the interference pattern and thereby also improves the accuracy of the measurements. The light exiting the etalon is focused by a Nikon $400 \mathrm{~mm}$ focal length lens (L6) onto the detector of a Princeton Instruments VersArray back-illuminated, scientific-grade CCD camera. The 512x512 imaging array has a $100 \%$ fill factor, $24 \times 24 \mu \mathrm{m}$ pixels, 16-bit dynamic range, and thermoelectric cooling and lownoise electronics. The images are acquired with a 5 second exposure time. Shorter exposure times are possible by using higher quantum efficiency detectors (i.e., photomultiplier tubes) and selecting optical components to maximize the optical throughput of the system. However, in this steady flow a long exposure time is applicable for demonstrating the technique. 


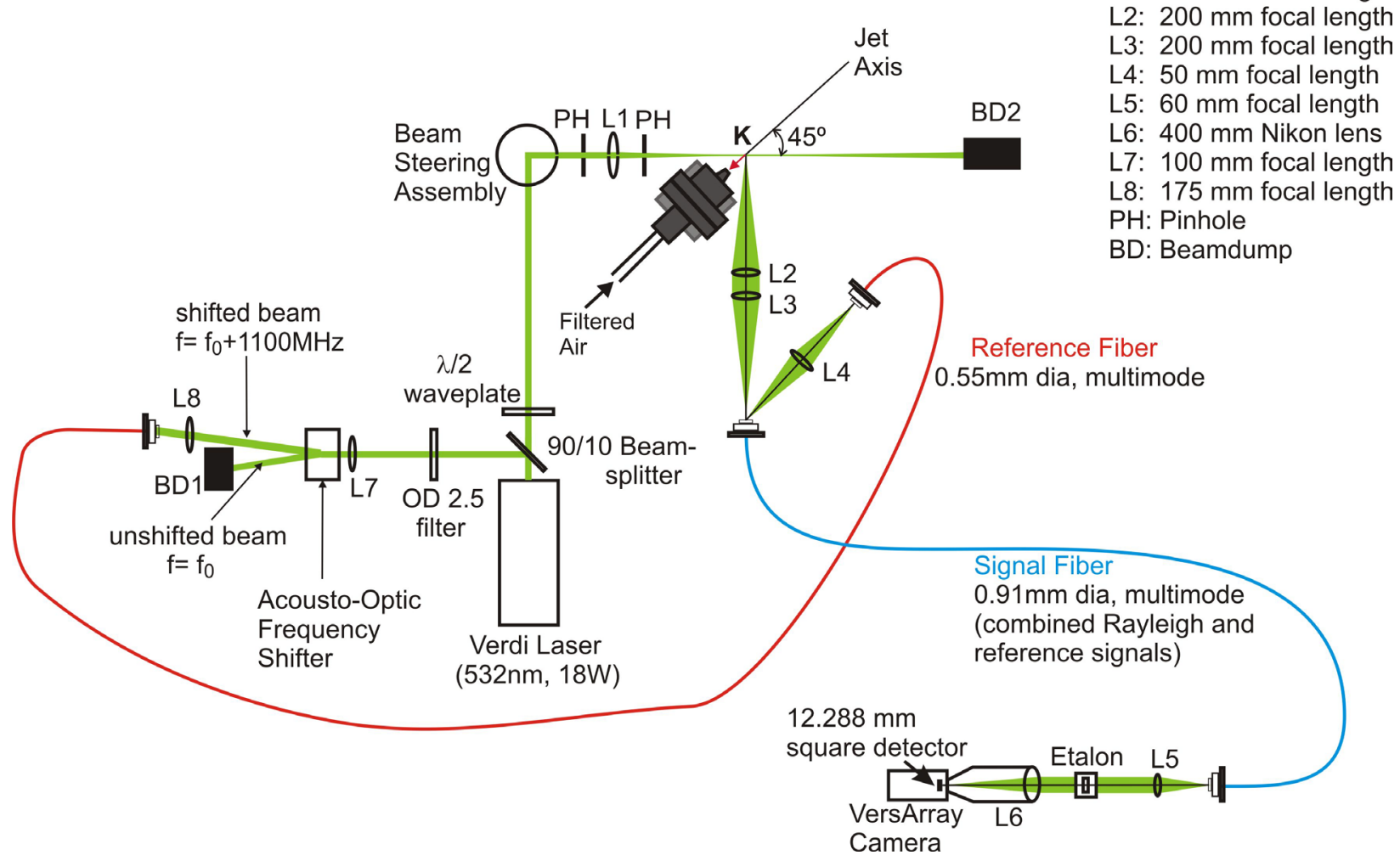

Fig. 8 Experiment schematic for the Rayleigh scattering experiment in a 10-mm diameter nozzle free jet flow at NASA GRC.

\section{Results}

\section{A. Calibration of Optical System Parameters}

Reference laser light at the original laser frequency is imaged along with laser light shifted upward in frequency by $1100 \mathrm{MHz}$ to calibrate the following optical system parameters: FSR and finesse of the Fabry-Perot etalon and the effective focal length $\left(f_{L}\right)$ of the Nikon camera lens (L6). Several images are acquired with the fringes located about half-way between the center of the fringe pattern and the edge of the image of the optical fiber face. The 0.91 $\mathrm{mm}$ diameter optical fiber is imaged with a magnification factor of 6.4 providing an imaged region of $5.83 \mathrm{~mm}$ diameter on the CCD detector. Beyond the extent of the image of the fiber face there is no light present and therefore no interference fringes are imaged. An image of the fiber face with white (broadband) light exiting the fiber is shown in Fig. 9 to illustrate the extent of the illuminated region of the image. A sample image containing shifted and unshifted reference light is shown in Fig. 10. The innermost interference ring is the unshifted reference light, the second ring as you move outward from center is the laser light that has been artificially shifted upward by $1100 \mathrm{MHz}$, the third ring is the illuminated cladding of the optical fiber, and lastly the next order of interference fringes (shifted and unshifted signals) are visible just beyond the fiber cladding due to the metal fiber holder reflecting some of the illumination into the system. 


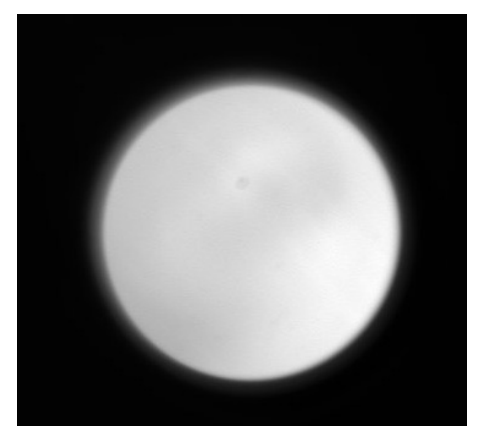

Fig. 9 The optical fiber face illuminated with a white light source imaged through the Fabry-Perot etalon onto the CCD detector. The diameter of the image of the fiber core is approximately $5.83 \mathrm{~mm}$.

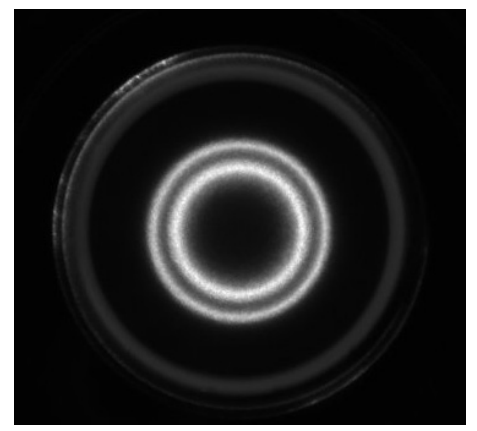

Fig. 10 Reference laser signal at the original laser frequency (inner fringe) and laser signal artificially shifted upward in frequency by $1100 \mathrm{MHz}\left(2^{\text {nd }}\right.$ fringe) imaged through the Fabry-Perot etalon. Images like this one were used to calibrate the finesse and free spectral range of the etalon.

A maximum likelihood estimation analysis is performed to fit the image data to a model function consisting of the addition of two Fabry-Perot instrument functions separated by $1100 \mathrm{MHz}$ and evaluate unknown parameters. This analysis results in the following values for the calibration constants based on averaging the results of four images:

$$
\begin{gathered}
\mathrm{FSR}=9.19 \mathrm{GHz} \\
\text { Finesse }=16.1 \\
f_{L}=402 \mathrm{~mm}
\end{gathered}
$$

Image data with both Rayleigh and shifted reference light signals combined in the interference pattern (as shown in Fig. 7) are used to calibrate the optical system efficiency factor $(\varepsilon)$. The Rayleigh signal was collected from a point on the jet centerline at an axial distance of two jet diameters. Data at the two highest flow velocities are used in this calibration. The efficiency factor is determined by assuming the flow parameters are equal to jet exit conditions based on isentropic flow and ideal gas law calculations, and fitting for the optical system efficiency by means of maximum likelihood estimation. Since the measurements are acquired within the jet core, this assumption should be reasonably accurate. By using the highest flow velocities, the Rayleigh and reference fringe peaks are at the maximum possible separation and the molecular number density is greatest, giving the maximum possible Rayleigh signal level. The resulting optical system efficiency factor is:

$$
\varepsilon=1.115 \%
$$

Now that these optical system factors are determined, the Rayleigh image data with shifted or unshifted reference light can be analyzed to determine the desired flow parameter estimates by maximum likelihood estimation.

\section{B. Nozzle flow results}

Rayleigh scattered light is collected from the measurement probe volume located on the centerline of the jet flow at an axial distance of two jet diameters downstream of the nozzle exit within the jet core. The flow velocity is varied from approximately $40 \mathrm{~m} / \mathrm{s}$ up to $190 \mathrm{~m} / \mathrm{s}$ over a temperature range of $279 \mathrm{~K}$ to $296 \mathrm{~K}$. At each flow condition Rayleigh image data are recorded first with artificially shifted reference light $\left(v_{\mathrm{ref}}=v_{0}+1100 \mathrm{MHz}\right)$ as in Fig. 7, and then with unshifted reference light $\left(v_{\text {ref }}=v_{0}\right)$ as in Fig. 6. The flow parameters are evaluated by maximum likelihood estimation using the model function previously developed (Eq. (11) of Ref. 14). The parameters that are evaluated in this process are the axial flow velocity, gas density, static temperature, reference signal amplitude, reference fringe radius, and the centroid of the circular interference pattern. Vertical and horizontal 


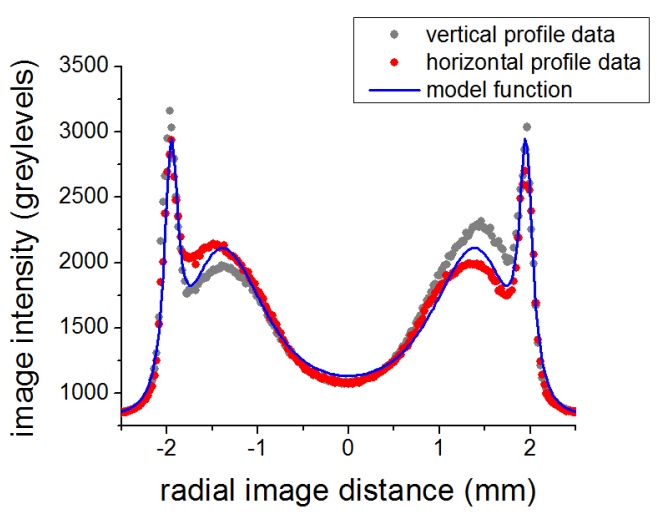

Fig. 11 Line profile through the center of an interference fringe image and the corresponding model function fit to the Rayleigh and artificially shifted $\left(v_{\text {ref }}=v_{0}+\right.$ $1100 \mathrm{MHz}$ ) reference signals for a flow velocity of $190 \mathrm{~m} / \mathrm{s}$.

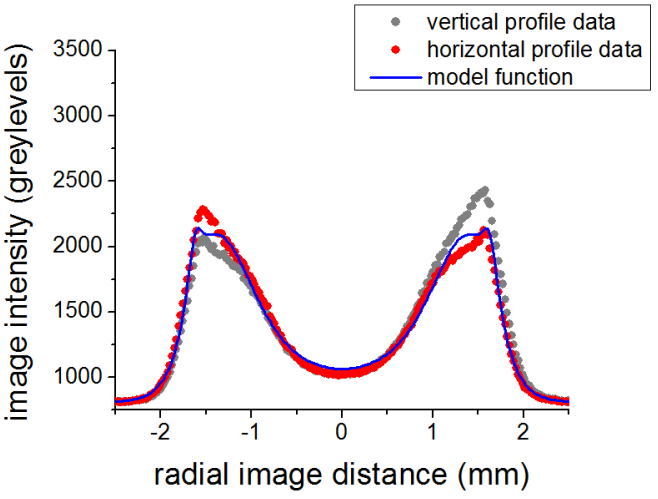

Fig. 12 Line profile through the center of an interference fringe image and the corresponding model function fit to the Rayleigh and unshifted $\left(v_{\text {ref }}=v_{0}\right)$ reference signals for a flow velocity of $190 \mathrm{~m} / \mathrm{s}$.

cross-sectional profiles through the interference fringes are plotted along with the model function fit to the image data for the highest flow velocity $(190 \mathrm{~m} / \mathrm{s})$ in Figs. 11 and 12 with shifted and unshifted reference light, respectively. These profiles show that the optical efficiency is not equal across the entire image as evidenced by varying amplitude of the fringe peaks. The varying efficiency is not taken into account in the analysis presented in this paper, however it will be looked at more closely in future work. If the efficiency at each pixel could be determined then the measurement accuracy would improve significantly, however this type of calibration is not a simple task. Another observation to make here is that the amplitude of the unshifted reference signal is much lower than the amplitude of the up-shifted reference signal. As the reference fringe overlaps the Rayleigh fringe more, less reference signal can be tolerated to maintain a useable Rayleigh fringe. Therefore the reference signal amplitude is only about 300 greylevels in the unshifted case, but the amplitude is about 1900 greylevels in the up-shifted case.

Plots of the resulting flow velocity, density, and temperature measurements are shown in Figs. 13-15, respectively. In each figure the calculated flow values (by way of isentropic flow and ideal gas equations) based on physical probe measurements of total and static pressure and total temperature are indicated by the blue curves. Measurements from the data containing artificially shifted reference signal are shown as black triangle symbols and measurements from data containing unshifted reference signal are shown as red circle symbols. All three flow parameter measurements are improved by having the reference signal up-shifted in frequency to spatially separate the Rayleigh and reference fringes. The two lowest velocity cases are unusable in the unshifted reference scenario because the Rayleigh and reference fringes did not have sufficient separation for the maximum likelihood analysis to converge on a solution. Even the measurements from the artificially shifted reference data have increased error at the lower velocities (which correspond to higher temperature and lower density cases) since the fringe separation is slightly less than in the higher velocity cases. The velocity, density, and temperature measurements are within $4 \mathrm{~m} / \mathrm{s}$, $1.5 \%$, and $11 \mathrm{~K}$, respectively, for the data with the reference frequency artificially shifted and velocities greater than $110 \mathrm{~m} / \mathrm{s}$. For the data with the unshifted reference signal the velocity measurements are, on average, about $85 \mathrm{~m} / \mathrm{s}$ too high, the densities are about $10 \%$ too low, and the temperatures are about 65 degrees too low. These errors would be unacceptable to most researchers attempting to study jet flows. The errors in the measurements where the reference signal frequency has been up-shifted are within an acceptable range for many situations and could be improved by shifting the frequency by an even greater amount and optimizing certain experiment parameters such as the amplitude of the reference signal. A Cramer-Rao lower bound (CRLB) uncertainty analysis was performed to demonstrate how the lower bound on the uncertainty in the flow measurements changes with the amount of artificial frequency shift imposed on the reference signal. The details of this type of analysis have been reported previously in Ref. 7 and Ref. 18. The results of the analysis in terms of velocity, temperature, and density measurement uncertainty as a function of artificial reference frequency shift are shown in Fig. 16 for a flow with a velocity of 190 $\mathrm{m} / \mathrm{s}$ and a temperature of $279 \mathrm{~K}$. The uncertainty in all flow parameters decreases as frequency shift increases up to $2 \mathrm{GHz}$, but the uncertainty levels flatten out beyond $2 \mathrm{GHz}$ and do not continue to improve beyond that point. 


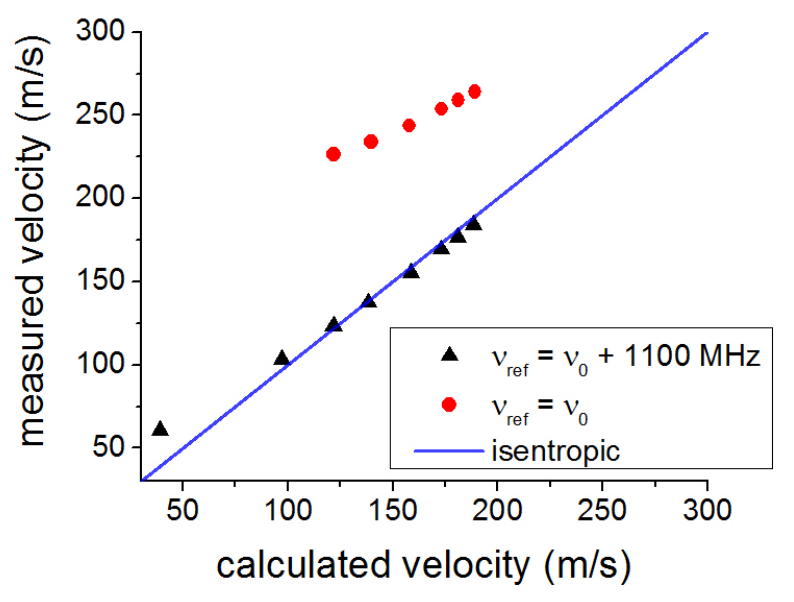

Fig. 13 Measured axial component of the flow velocity versus actual (calculated) values for Rayleigh data containing shifted and unshifted reference laser signal.

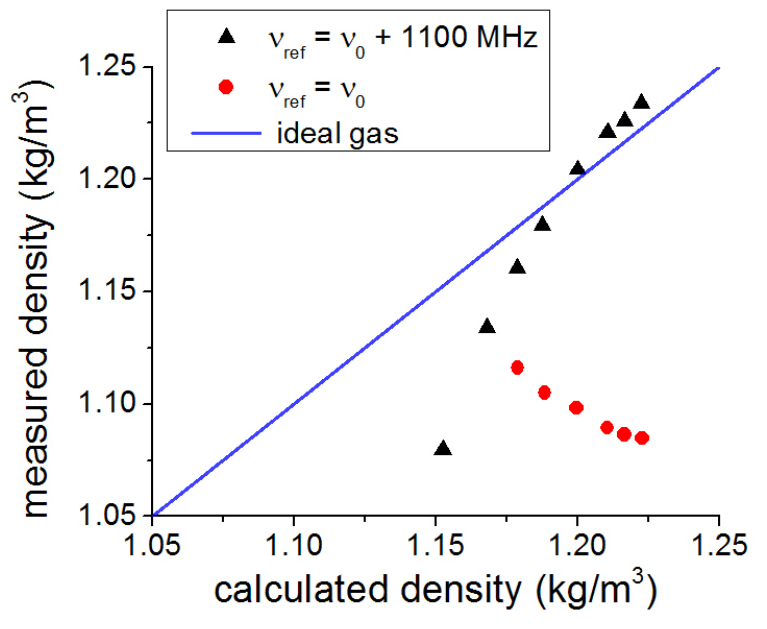

Fig. 14 Measured gas density versus actual (calculated) values for Rayleigh data containing shifted and unshifted reference laser signal.

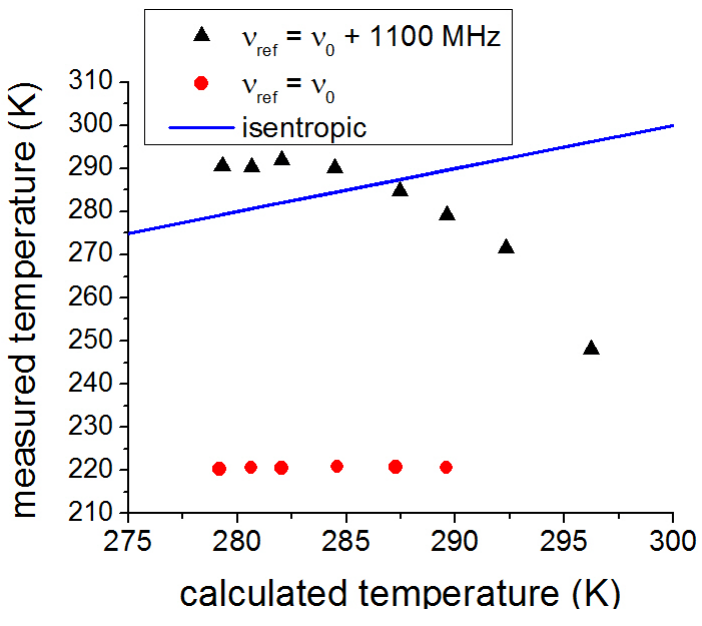

Fig. 15 Measured gas temperature versus actual (calculated) values for Rayleigh data containing shifted and unshifted reference laser signal.

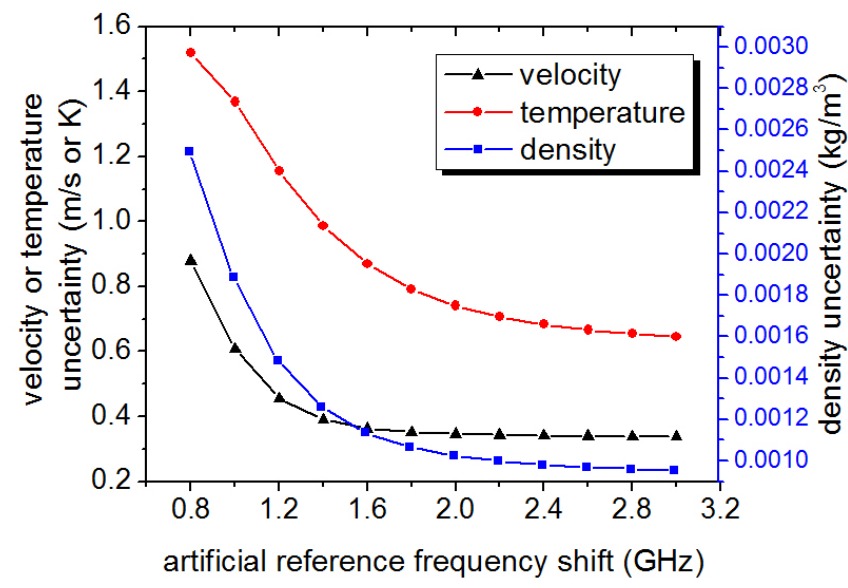

Fig. 16 CRLB uncertainty analysis of velocity, temperature, and density measurement uncertainty as a function of artificial reference frequency shift.

\section{Conclusions and Future Work}

An acousto-optic frequency shifting device is evaluated as a tool for use in spectroscopic Rayleigh scattering experiments. The frequency shifting device provides a means of shifting the incident or reference laser frequency by $1100 \mathrm{MHz}$ to avoid overlap of the Rayleigh and reference signal peaks in the interference pattern used to obtain the velocity, density, and temperature measurements, and also to calibrate the free spectral range of the Fabry-Perot etalon used in the spectroscopic measurements. A Rayleigh scattering system using the frequency shifting device to impose an artificial shift in the reference signal of $1100 \mathrm{MHz}$ is applied in a $10 \mathrm{~mm}$ diameter subsonic nozzle flow to evaluate the measurement accuracy improvement compared to a system utilizing unshifted reference light coupled with the Rayleigh signal. The improvements in measurement accuracy are significant. The data containing unshifted light have extremely high and unacceptable measurement errors whereas the data containing artificially frequency- 
shifted reference signal have errors of $4 \mathrm{~m} / \mathrm{s}$ or better in velocity, $1.5 \%$ or better in density, and $11 \mathrm{~K}$ or better in temperature for velocities greater than $110 \mathrm{~m} / \mathrm{s}$. At lower velocities the measurement errors are higher because the spatial shift between the reference and Rayleigh fringe peaks are not sufficient due to the lower Doppler shift associated with those velocity levels. The measurement accuracy can be improved further by increasing the artificial shift in the reference frequency. The device used here provides a maximum shift of $1100 \mathrm{MHz}$; however an acoustooptic frequency shifter providing a shift of at least $2000 \mathrm{MHz}$ would be optimal since a lower bound uncertainty analysis demonstrated this as the frequency shift resulting in minimum measurement uncertainty. An additional improvement for future work is to determine a method for calibrating the optical efficiency across the image to improve the model function used to estimate the flow parameters. The use of an acousto-optic device for reference frequency shifting would be especially valuable for Rayleigh experiments using pulsed laser systems where the frequency can vary significantly from shot-to-shot and must be measured simultaneously with the Rayleigh signal.

\section{Acknowledgments}

The authors would like to thank Professor Tenti of the University of Waterloo for providing the TENTI S6 numerical code for calculating the Rayleigh spectrum. This work is supported by the Supersonics and Subsonic Fixed Wing Projects under NASA's Fundamental Aeronautics Program.

\section{References}

${ }^{1}$ Miles, R. B., Lempert, W. R., and Forkey, J. N., “Laser Rayleigh Scattering,” Meas. Sci. Technol., Vol. 12, No. 5, 2001, pp. R33-R51.

${ }^{2}$ Forkey, J. N., Lempert, W. R., and Miles, R. B., “Accuracy Limits for Planar Measurements of Flow Field Velocity, Temperature, and Pressure Using Filtered Rayleigh Scattering,” Exp. Fluids, Vol. 24, No. 2, 1998, pp. 151-162.

${ }^{3}$ Boguszko, M., and Elliott, G. S., "On the Use of Filtered Rayleigh Scattering for Measurements in Compressible Flows and Thermal Fields," Exp. Fluids, Vol. 38, No. 1, 2005, pp. 33-49.

${ }^{4}$ Vaughan, J. M., The Fabry Perot Interferometer, History, Theory, Practice, and Applications, Adam Hilger, Philadelphia, 1989, pp. 89-134.

${ }^{5}$ Panda, J., and Seasholtz, R. G., "Velocity and Temperature Measurement in Supersonic Free Jets Using Spectrally Resolved Rayleigh Scattering," AIAA-99-0296, 1999.

${ }^{6}$ Seasholtz, R. G., Panda, J., and Elam, K. A., "Rayleigh Scattering Diagnostic for Measurement of Velocity and Density Fluctuation Spectra," AIAA-2002-0827, 2002.

${ }^{7}$ Mielke, A. F., Elam, K. A., and Sung, C. J., "Multiproperty Measurements at High Sampling Rates Using Rayleigh Scattering," AIAA Journal, Vol. 47, No. 4, 2009, 850-862.

${ }^{8}$ Seasholtz, R. G., Buggele, A. E., and Reeder, M. F., "Flow Measurements Based on Rayleigh Scattering and Fabry-Perot Interferometer," Optics and Lasers in Engineering, Vol. 27, No. 6, 1997, pp. 543-570.

${ }^{9}$ Bivolaru, D., Danehy, P. M., Gaffney, R. L., and Cutler, A. D., "Direct-View Multi-Point Two-Component Interferometric Rayleigh Scattering Velocimeter," AIAA-2008-236, 2008.

${ }^{10}$ Lock, J. A., Seasholtz, R. G., and John, W. T., "Rayleigh-Brillouin Scattering to Determine One-Dimensional Temperature and Number Density Profiles of a Gas Flow Field," Applied Optics, Vol. 31, No. 15, 1992, pp. 2839-2848.

${ }^{11}$ Mielke-Fagan, A. F., Elam, K. A., and Clem, M. M., "Multiple-Point Mass Flux Measurement System Using Rayleigh Scattering," AIAA-2009-528, 2009.

${ }^{12}$ Mielke, A. F., Seasholtz, R. G., Elam, K.A., and Panda, J., “Time-average Measurement of Velocity, Density, Temperature, and Turbulence Velocity Fluctuations Using Rayleigh and Mie Scattering," Experiments in Fluids, Vol. 39, No. 2, 2005, 441454.

${ }^{13}$ Mielke-Fagan, A. F., Clem, M. M., Elam, K. A., and Hirt, S. M., "Progress on a Rayleigh Scattering Mass Flux Measurement Technique," AIAA-2010-856, 2010.

${ }^{14}$ Mielke-Fagan, A. F., Clem, M. M., Elam, K. A., "Rayleigh Scattering Measurements Using a Tunable Liquid Crystal Fabry-Perot Interferometer," AIAA-2010-4350, 2010.

${ }^{15}$ Tenti, G., Boley, C. D., and Desai, R. C., "On the Kinetic Model Description of Rayleigh-Brillouin Scattering from Molecular Gases," Canadian Journal of Physics, Vol. 52, No. 4, 1974, pp. 285-290.

${ }^{16}$ Boley, C. D., Desai, R. C., and Tenti, G., "Kinetic models and Brillouin scattering in a molecular gas," Canadian Journal of Physics, Vol. 50, No. 18, 1972, pp. 2158-2173.

${ }^{17}$ Edwards, R. V., Processing Random Data: Statistics for Engineers and Scientists, World Scientific, New Jersey, 2006, pp.91-97.

${ }^{18}$ Mielke, A. F., "Development of a Molecular Rayleigh Scattering Diagnostic for Simultaneous Time-Resolved Measurement of Temperature, Velocity, and Density," Ph.D. Dissertation, Department of Mechanical and Aerospace Engineering, Case Western Reserve Univ., Cleveland, OH, 2008, pp. 170-176. 